New

Methods

\title{
A Novel Sizing Technique for Mitral Annuloplasty
}

Nobuhisa Ohno, MD, PhD, Toshi Maeda, MD, Otohime Kato, MD, Go Ueno, MD,

Kosuke Yoshizawa, MD, and Keiichi Fujiwara, MD, PhD

\begin{abstract}
Although sizing for mitral annuloplasty (MAP) is a critical part of mitral valve repair, no consistent method is used, and precise size adjustment is done somewhat by intuition. We developed a new original ring size tester and have been applying it to our mitral valve repair procedure. The concept is that the tester shapes the annulus into exactly the same size and form as the target annuloplasty while confirming a good leaflet coaptation with a saline test to simulate annuloplasty. This original tester provides the best ring size for MAP that is neither too large nor too small. In this paper, we introduce our original ring size tester and describe how it is used for MAP.
\end{abstract}

Keywords: mitral annuloplasty, ring size tester, systolic anterior motion, functional mitral stenosis

\section{Introduction}

In most mitral valve repair procedures, mitral annuloplasty (MAP) with ring is combined with leaflet repair. The purpose of the MAP is to secure enough leaflet coaptation and prevent re-dilatation of the annulus that affects valve durability. ${ }^{1)}$ The procedure of sizing is very critical, because too large size selection may cause an incompetent valve, and too small size selection may cause systolic anterior motion (SAM) or functional mitral stenosis (FMS). ${ }^{2-4)}$ At present, various types of rings have been introduced by manufacturers, and they provide a specific sizer for each product. Basically, those sizers have a D-shaped template with two notches. The dimensions of the template and

Department of Cardiovascular Surgery, Hyogo Prefectural Amagasaki General Medical Center, Amagasaki, Hyogo, Japan

Received: January 4, 2019; Accepted: February 21, 2019 Corresponding author: Nobuhisa Ohno, MD, PhD. Department of Cardiovascular Surgery, Hyogo Prefectural Amagasaki General Medical Center, 2-17-77 Higashinaniwa-cho, Amagasaki, Hyogo 660-8550, Japan

Email: nobcvs@mac.com

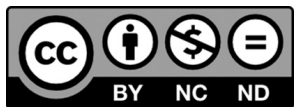

This work is licensed under a Creative Commons Attribution-NonCommercialNoDerivatives International License.

(C)2019 The Editorial Committee of Annals of Thoracic and Cardiovascular Surgery inter-notch distance are used for ring sizing. Methods of sizing are usually described in the implant manual provided by the manufacturer, but they vary greatly among products. Some products recommend anterior leaflet area, others specify anterior leaflet height, inter-commissural distance, inter-trigonal distance, etc. Moreover, measuring the area or the distance by placing a certain shaped platform to the floppy native valve is sometimes uncertain and depends on the surgeon's experience and intuition. There is no scientific approach to the current sizing process. ${ }^{5}$ Based on this background, we developed a new ring size tester (RST) for accurate and reproducible annular sizing, and we have had good results with the new sizing technique.

\section{Methods}

The new RST consisted of two large (outer) and small (inner) metal rings attached to a metal handle. The shape of the rings was like annuloplasty rings when used. Sizes of these metal rings were set so that there was a 1-mm gap between them, and the outline of the gap was equal in size to the matched labeled annuloplasty ring. The outer ring has a small $(1 \mathrm{~mm})$ opening at the 6 o'clock position. The labeled size was from 24 to 40 , and the anteroposterior inner diameter was from $15.6 \mathrm{~mm}$ to $27.9 \mathrm{~mm}$, respectively (Fig. 1). In this series, the labeled size and the antero-posterior diameter were like those of 


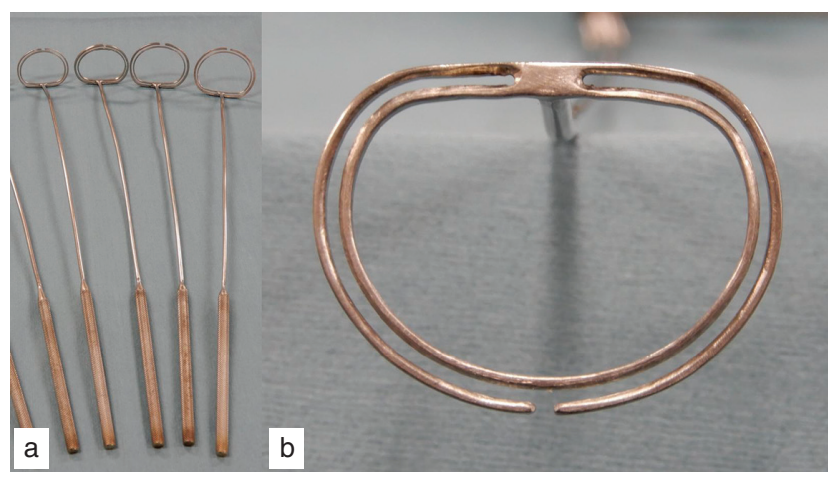

Fig. 1 Group sets of a new ring size tester (a). The head part of the tester consists of two metal rings with a $1 \mathrm{~mm}$ gap between them with a small $(1 \mathrm{~mm})$ opening at the 6 o'clock position in the outer ring (b).

Carpentier-Edwards (CE) Physio II annuloplasty ring (Edwards Lifesciences, Irvine, CA USA), but this device could be used for other types of rigid or semi-rigid rings if their antero-posterior diameter was pre-measured.

After mitral valve exposure, 12 to 14 2-0 braided polyester U-shaped stay sutures for MAP were placed in the mitral annulus. Leaflet plasty such as expanded polytetrafluoroethylene (ePTFE) chordal placement for prolapse and/or small leaflet resection for thick excessive tissue was performed using the normal procedure before sizing. Dots were placed 8 to $10 \mathrm{~mm}$ from the free edges of the leaflets with a skin marker pen to highlight the coaptation line. First, a rough estimate of the size was made with commercial sizers. Then, the estimated size from the new RST set was chosen. The tester was placed in the left atrium while annuloplasty sutures were slipped in the gap though the opening of the outer ring of the tester. The tester was pushed on the annulus, and sutures were pulled to maintain tension. Annuloplasty was simulated by this maneuver. Then, the saline test was performed using the normal procedure to check the coaptation pattern (Fig. 2). If ink dots were not identified and far down in the left ventricular side, a larger RST was tried. If a rough zone was seen with ink dots, the coaptation length was too short, and a smaller RST was applied. The procedure was repeated until the best coaptation, neither too much nor too little, was obtained. In our experience, one or two upsized rings could be selected in some patients compared to a commercial sizer.

Written informed consent was obtained from the patient for the procedure and the publication of this report and accompanying images. The Institutional Review Board of the Hyogo Prefectural Amagasaki General Medical Center approved the use of this device.

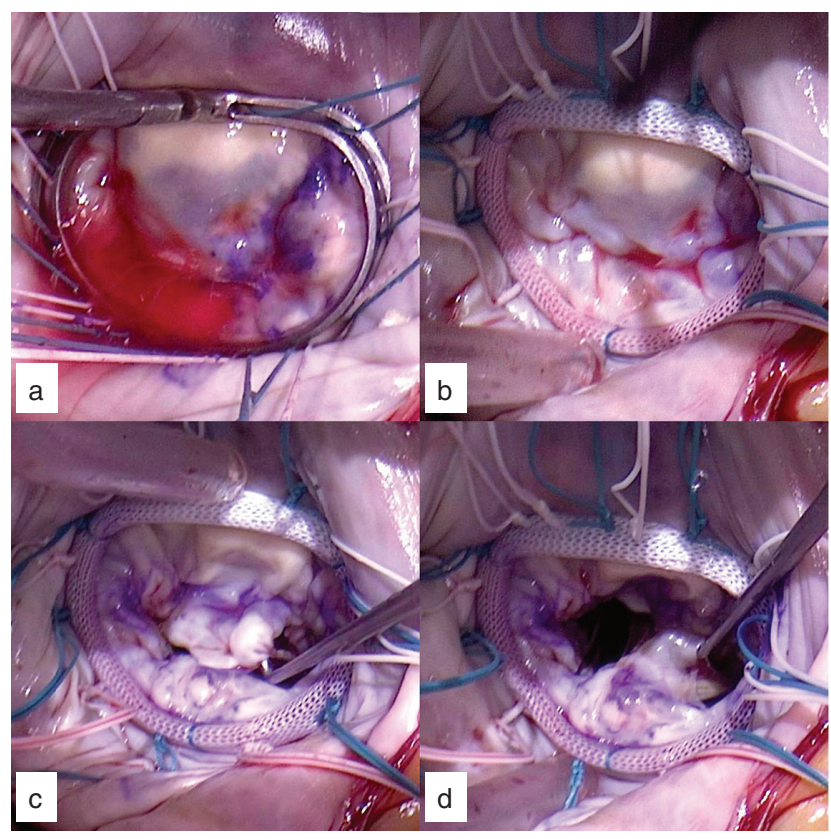

Fig. 2 Annular ring size determination with a new ring size tester. (a) Saline test using the tester as if the annulus is corrected in the form as when annuloplasty is performed. (b) Final result of mitral annuloplasty. Good coaptation margin of the anterior (c) and the posterior leaflet (d) are obtained.

\section{Results}

This technique has been applied in 20 patients with degenerative mitral valve disease since January 2014. Concomitant procedures consisted of tricuspid valve repair $(n=6)$, atrial ablation $(n=5)$, coronary artery bypass grafting $(n=3)$, aortic valve replacement $(n=1)$, and ascending aortic replacement $(n=1)$. Minimally invasive approach via right mini-thoracotomy was adopted in one patient. Mean cardiopulmonary bypass time was $205 \pm 47$ minutes, and myocardial ischemic time was $159 \pm 39$ minutes. Leaflet repair was done only for the anterior leaflet $(n=3)$, only for the posterior leaflet $(n=10)$, and for both leaflets $(n=5)$. Isolated MAP was done for two patients. Repair technique was triangular leaflet resection in $15(\mathrm{P} 1: \mathrm{n}=2, \mathrm{P} 2: \mathrm{n}=6, \mathrm{P} 3: \mathrm{n}=7)$ and chordal replacement with ePTFE sutures in 12 (A2: $n=7, A 3: n=4, P 1: n=3, P 2: n=5, P 3: n=1)$. CE Physio II was used in all procedures, and the ring size was $31.0 \pm 2.3$. Five patients had two upsized rings, nine patients had one upsized rings, and six patients had the same size of rings estimated by commercial sizer. There was no mortality, or any major complication. Mitral valve area measured $2.57 \pm 0.5 \mathrm{~cm}^{2}$, pressure gradient at rest was $2.5 \pm 1.3 \mathrm{mmHg}$, and coaptation length was 
$8.8 \pm 2.1 \mathrm{~mm}$ by transthoracic echo at discharge. All the patients have been well with minimal or no mitral valve leakage so far.

\section{Discussion}

A variety of annuloplasty rings are made by different manufacturers. These rings are designed for the specific disease type based on different concepts. Sizing technique is shown in the implant manual provided by the manufacturer or written in the literature. However, the process may be confusing, because some authors have introduced a sizing process different from the implant manual. ${ }^{5)}$ It is suggested that surgical technique should be scientifically determined, and the technique should be clearly described, especially for young surgeons.

When the ring size is determined, the surgeon takes great care with the coaptation area. Even if the selected ring is smaller, it results in an increased coaptation area with minimal risk of valve insufficiency. However, it is reported that excess downsizing may cause not only SAM but also FMS on exercise. ${ }^{2-4)}$ Thus, the optimal ring size is essential. The new sizing technique described in this study enables surgeons to simulate MAP while performing the leak test. If the coaptation area seems too large, the test can be repeated with a larger tester until the optimal size is determined.

Anyanwu and Adams reported the "ink test" as an assessment tool in mitral valve repair. ${ }^{6)}$ In the technique, the closing line is marked from the anterior to the posterior commissure with a surgical marking pen, while the mitral valve is closed in a systolic position by the saline test. Then, saline is removed from the ventricle, following an inspection of each leaflet coaptation. In the new technique, the closing line is also marked as some dots with marking pen, but the concept is somewhat different. The dots are marked beforehand to ensure adequate coaptation area followed by the saline test and fine-tuning. The confirmation style in the new method is like the technique introduced in the American correction by Lawrie. ${ }^{7}$

In this series of annuloplasties, up to three different sizes of RST were used in the saline test. Annuloplasty sutures must be slipped in the slit of the RST, which takes around a minute for each test. We think that the extra time for the process is acceptable, and the benefit of our new method should be immeasurable.

We applied this device for CG Future (CG) annuloplasty ring (Medtronic, Minneapolis, MN, USA) in three cases after this series. We measured antero-posterior diameter of each size of the CG ring and the device. Then, selected the ring size by the diameter matched to the determined device size. Although we had good results for CG ring as well, further studies are needed to prove the versatility of the device.

As a limitation of this device, both the outer and inner rings are flat in shape. Three-dimensional shape is popular for the modern annuloplasty rings, and this device might not completely imitate the annuloplasty. However, the main purpose of this device is to confirm the coaptation amount and pattern, and three-dimensional shape might not much influence over the results. Future modifications of its design are awaited.

\section{Conclusion}

A new RST was developed for MAP with the aim of a more scientific approach. The RST enabled the surgeon to accomplish consistent and reproducible mitral valve repair. This device and technique should be very useful for mitral valve repair, not only for young surgeons with less experience but also for all experienced surgeons.

\section{Disclosure Statement}

All authors have no conflict of interest.

\section{References}

1) Carpentier AF, Lessana A, Relland JY, et al. The "physio-ring": an advanced concept in mitral valve annuloplasty. Ann Thorac Surg 1995; 60: 1177-85.

2) Loulmet DF, Yaffee DW, Ursomanno PA, et al. Systolic anterior motion of the mitral valve: a 30-year perspective. J Thorac Cardiovasc Surg 2014; 148: 2787-93.

3) Mesana TG, Lam BK, Chan V, et al. Clinical evaluation of functional mitral stenosis after mitral valve repair for degenerative disease: potential affect on surgical strategy. J Thorac Cardiovasc Surg 2013; 146: 1418-23.

4) Doi K, Yamano T, Ohira S, et al. Annuloplasty ring size determines exercise-induced mitral stenosis severity after valve repair. J Heart Valve Dis 2015; 24: 744-51.

5) Bothe W, Miller DC, Doenst T. Sizing for mitral annuloplasty: where does science stop and voodoo begin? Ann Thorac Surg 2013; 95: 1475-83.

6) Anyanwu AC, Adams DH. The intraoperative "ink test": a novel assessment tool in mitral valve repair. J Thorac Cardiovasc Surg 2007; 133: 1635-6.

7) Lawrie GM. Ensuring proper leaflet apposition during mitral valve repair. J Thorac Cardiovasc Surg 2008; 135: 228. 